\title{
Quantifying the effects of standing waves within the skull for ultrasound mediated opening of the blood-brain-barrier
}

\author{
Eleanor Martin \\ University College London \\ London, UK \\ elly.martin@ucl.ac.uk
}

\author{
Andrew Hurrell \\ Precision Acoustics Ltd. \\ Dorchester, UK \\ 0000-0002-3477-128X
}

\author{
James Choi \\ Imperial College London \\ London, UK \\ j.choi@imperial.ac.uk
}

\author{
Bradley Treeby \\ University College London \\ London, UK \\ b.treeby@ucl.ac.uk
}

\begin{abstract}
Ultrasound mediated opening of the blood-brain barrier (BBB) has been shown to be effective in enhancing the delivery of therapeutic agents to the brain. However, challenges remain in targeting and specificity of $\mathrm{BBB}$ opening due to attenuation, aberration and reverberation of transcranial ultrasound fields. In this study, experimental and numerical assessment was performed of standing waves within an ex vivo human skull when delivering ultrasound pulses of varying lengths at $300 \mathrm{kHz}$ using a large aperture focused ultrasound transducer. Simulations showed minimal distortion of the focal region but low amplitude standing waves were established within the skull with bursts of 50 cycles or more. Under the same conditions, the experimental measurements showed small variations in focal pressure which took 300 to $600 \mu$ s to stabilise. The pattern of sidelobes and superimposed standing waves was generally more complex when the focus was placed closer to the side and base of the skull. This data supports the use of large aperture diameter transducers and short pulse lengths for targeted BBB opening.
\end{abstract}

Index Terms-blood-brain barrier, focused ultrasound, standing waves

\section{INTRODUCTION}

Significant advances have been made in the development of therapeutic agents for the treatment of neurodegenerative diseases and brain cancers. However, the blood-brain barrier (BBB) presents a major impediment to the delivery of larger molecules into the interstitial fluid of the brain, which severely limits the clinical efficacy of these agents [1]. There is now well-established evidence that ultrasound, when combined with microbubbles, can reversibly and selectively disrupt the BBB [2], [3]. One remaining challenge in the transcranial application of ultrasound is that the skull can lead to significant attenuation and aberration of the transmitted waves, which affects the quality of ultrasound focusing [4], and thus the targeting and specificity of the BBB opening. This can be mitigated using lower frequency ultrasound waves (below 500 $\mathrm{kHz}$ ), however, this increases the presence of standing waves, which can have a similarly deleterious effect on treatment specificity. However, the formation of standing waves can be reduced by using larger aperture diameter transducers [5]. The use of short pulse lengths can also reduce standing waves, and

This work was supported by the ThUNDDAR EPSRC Network Plus. recent work has demonstrated that they can achieve effective opening of the BBB and uniform delivery of therapeutic agents [6]. The aim of this study was to examine the formation of standing waves within the skull with a large transducer aperture diameter coupled with different ultrasound pulse lengths.

\section{TRANSDUCER DESIGN AND CHARACTERISATION}

To effectively sonicate deep brain structures such as the thalamus and the hippocampus, a transducer with a sufficient focal length is required. Given that the average size of a human head is $19 \mathrm{~cm}$ long and $15 \mathrm{~cm}$ wide [7], then allowing for acoustic coupling, a transducer focal length (i.e. radius of curvature) of $100 \mathrm{~mm}$ was selected. Given a fixed focal length, the length and width of the focal zone scale inversely with the aperture diameter of the transducer. A larger aperture diameter reduces the focal size and the effect of standing waves [5], but also increases fabrication cost and makes acoustic coupling more difficult. Balancing these two factors, an aperture diameter of $105 \mathrm{~mm}$ was therefore selected.

A custom transducer was fabricated with the chosen dimensions from a 1-3 piezocomposite of PZT and resin with an electrical matching network located within the transducer housing. The $-6 \mathrm{~dB}$ bandwidth range was $260 \mathrm{kHz}$ to $600 \mathrm{kHz}$ (fractional bandwidth $80.5 \%$ ) with maximum output at 470 $\mathrm{kHz}$ and a secondary peak at $300 \mathrm{kHz}$. A driving frequency of $300 \mathrm{kHz}$ was chosen for investigation of the beam stability inside the skull in order to reduce transcranial aberration and attenuation [8]. Characterisation, intra-skull measurements, and simulations were all performed at this frequency.

To characterise the acoustic field generated by the transducer in free-field, it was driven with a 12-cycle sinusoidal burst generated by an arbitrary waveform generator (33522A, Agilent, Berkshire, UK) amplified by a $300 \mathrm{~W}$ RF power amplifier (A300, Electronics and Innovation Ltd., Rochester, NY, USA). The peak-to-peak drive voltage measured at the end of the transducer cable was $12.7 \mathrm{~V}$, which was sufficiently low to ensure that the acoustic field contained no significant energy at the harmonic frequencies. The transducer was mounted in a fixed position using opto-mechanical components and a 
custom laser-cut Perspex mount in an automated scanning tank filled with degassed, deionised water. The acoustic pressure was measured with a calibrated $0.2 \mathrm{~mm}$ PVDF needle hydrophone (Precision Acoustics, Dorchester, UK) mounted and positioned with a 3-axis (X, Y, Z) computer-controlled translation stage. Waveforms were acquired, digitised and stored via a digital phosphor oscilloscope (DPO5034B, Tektronix UK Ltd., Berkshire, UK).

During measurements, the water temperature was $18.9^{\circ} \mathrm{C}$. The hydrophone was aligned to the position of spatial-peak pressure and the axial position was calculated from the timeof-flight of the ultrasound wave at the focus. Axial and lateral line scans passing through the focus were acquired with 0.5 $\mathrm{mm}$ steps. Waveforms were acquired at a sample rate of 62.5 $\mathrm{MHz}$ with 32 averages, in a time window that included approximately 5 cycles of the toneburst after the ring up period, which was approximately 5 cycles long. The acquired voltage waveforms, were windowed and filtered before the frequency response of the hydrophone was deconvolved to obtain the acoustic pressure. The amplitude of the FFT of the pressure waveforms at $300 \mathrm{kHz}$ was then extracted. The focus was at a distance of $92.5 \mathrm{~mm}$ from the transducer, the lateral $-6 \mathrm{~dB}$ focal width was $6 \mathrm{~mm}$, and the axial length ($6 \mathrm{~dB}$ ) of the focus was $49 \mathrm{~mm}$. Focal waveforms were also acquired with bursts of length 1, 5, 10, 50, 100 and 500 cycles for comparison with intra-skull measurements.

\section{EXPERIMENTAL MEASUREMENT OF TRANS-CRANIAL WAVEFORMS}

\section{A. Methods}

To assess the effects of standing waves within the skull, acoustic waveforms generated by the constructed transducer were measured at 2 positions inside an ex vivo human skull obtained under a material transfer agreement in accordance with the UK Human Tissue Act. Prior to the experiments, the skull was placed in a container filled with deionised water and degassed for 48 hours at -400 mbar. The skull was then positioned in the scanning tank using a laser cut Perspex mount and optomechanical components. The skull was inverted so that the foramen magnum (located in the base of the skull) was at the top, with the back of the skull oriented towards the transducer as shown in Fig. 1. The hydrophone was mounted in a fixed (rather than scanned) configuration using optomechanical components. In the first measurement position, the hydrophone needle was mounted vertically through the foramen magnum so that the sensitive element was placed centrally in the skull cavity, at a $90^{\circ}$ angle to the beam axis. The transducer was mounted on the xyz translation arm and the position was adjusted to maximise the measured waveform amplitude at the hydrophone. This was achieved by scanning the transducer in the axial and lateral directions at the back of the skull until the maximum was located. In the second position, the hydrophone was angled at approximately $45^{\circ}$ to the vertical, so the sensitive element was displaced lateral to the beam axis resulting in a position closer to the side and the base of the skull. The transducer was then
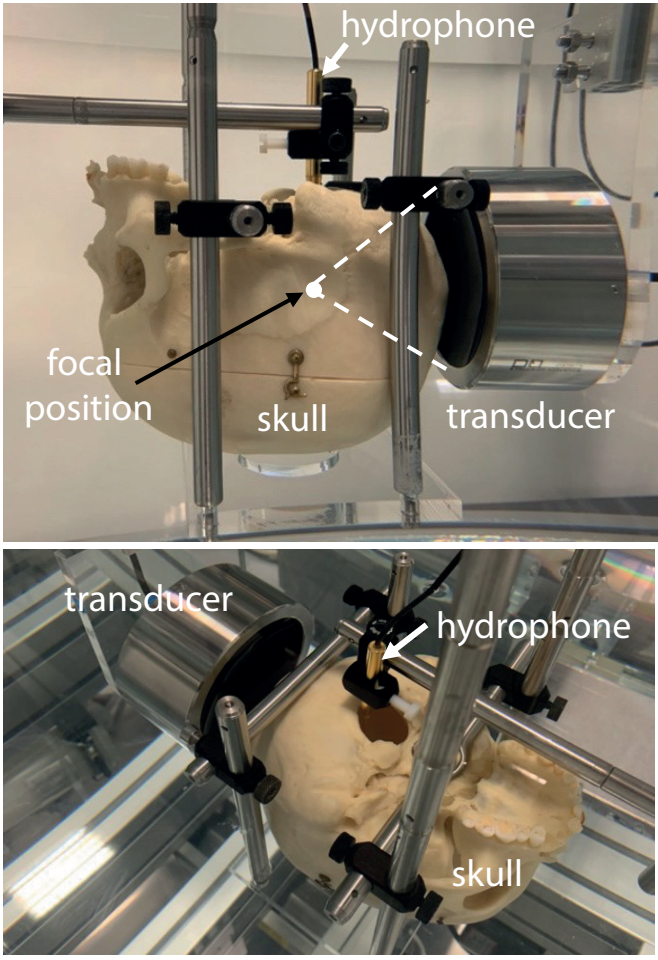

Fig. 1. Experimental set up showing ex vivo human skull mounted in scanning tank with hydrophone positioned through the foramen magnum. The transducer is aligned with the focus coincident with the hydrophone tip.

realigned to maximise the pressure at the hydrophone, which resulted in a translation of $22 \mathrm{~mm}$ laterally (away from the midline) and $11 \mathrm{~mm}$ inferiorly (towards the skull base).

At each position inside the skull, the transducer was driven with bursts of length 1, 5, 10, 50, 100 and 500 cycles, with a peak-to-peak drive voltage of $73.9 \mathrm{~V}$, which resulted in a similar focal amplitude to that measured in free-field. Waveforms were acquired with the needle hydrophone with an additional booster amplifier to allow inspection of reflections, with 1000 averages, in a $1.6 \mathrm{~ms}$ time window, which was long enough to contain the 500-cycle burst. The sensitivity of the hydrophone plus booster amplifier at $300 \mathrm{kHz}$ was derived by comparison of free-field focal waveforms acquired with and without the booster amplifier. Acquired waveforms were windowed and filtered, the complex frequency response of the hydrophone was deconvolved, and the waveforms were scaled to account for the sensitivity of the booster amplifier. The angle-dependent sensitivity of the hydrophone was neglected as the change in sensitivity between normal incidence and 90 degrees is less than $1 \%$ at $300 \mathrm{kHz}$, based on a plane piston model of hydrophone directivity.

\section{B. Results}

The insertion loss of the skull was calculated from the ratio the of focal pressure amplitude at each position within the skull and the focal pressure amplitude in free field. The magnitude of the acquired waveforms at the driving frequency was extracted from a steady part of the waveform. At position 



Fig. 2. Envelopes of measured (top) and simulated (bottom) 500 cycle waveforms at the focus in free-field, at position 1 central in the skull cavity, and at position 2, laterally and inferiorly offset from the skull centre.

1, the insertion loss was $-11.4 \mathrm{~dB}$ and at position 2 it was $-10.0 \mathrm{~dB}$. These measurements agree well with insertion loss measurements performed in [9], where the average insertion loss at $270 \mathrm{kHz}$ was measured to be $-9.8 \mathrm{~dB}$.

The stability of the beam at the position of peak pressure was examined by taking the envelope of the signal recorded when using 500 cycles. These are shown in Fig. 2. In freefield, it takes approximately $200 \mu$ s for the small undulations in the envelope to stabilise. This is increased to approximately $600 \mu \mathrm{s}$ and $500 \mu \mathrm{s}$ for position 1 and position 2 within the skull. This corresponds to the time window over which reflections were seen following the shorter bursts. However, the reflections were much smaller than the main pulse, and the later undulations in the beam are also small compared to the steady state pressure.

\section{Simulation OF TRANS-CRANIAL FIELDS}

\section{A. Methods}

A series of numerical simulations were performed to replicate the experimental study and to further obtain the complete
3D pressure field inside the skull. Simulations were conducted using kspaceFirstorder3D within the open-source kWave toolbox (V1.3) [10]. The source geometry was defined using an off-grid source with the nominal transducer parameters reported in Sec II [11]. To define the skull geometry, X-ray CT images of the skull were obtained using a Siemens SOMATOM Force CT scanner using a Head Spiral protocol with Hr59 bone convolution kernel. The acquisition settings were: $1000 \mathrm{~ms}$ exposure time, $100 \mathrm{kV}$ peak tube voltage, 45 $\mathrm{mA}$ tube current, $0.8 \mathrm{~mm}$ focal spot size, $1 \mathrm{~mm}$ slice thickness, $1 \mathrm{~mm}$ slice separation, $0.4 \mathrm{~mm}$ in plane pixel resolution. The CT images were imported into MATLAB then the CT Hounsfield units were converted to density, sound speed, and attenuation maps using a porosity mixture model as outlined in [12]. The medium maps were then resampled to give 6 grid points per wavelength at $300 \mathrm{kHz}$. The domain size was set to cover the complete skull and transducer, giving a grid size of $178 \times 268 \times 220$ grid points without the PML. The simulations were run for $1.6 \mathrm{~ms}$ with a CFL number of 0.05 (57k time steps). The simulations were performed using the native CUDA GPU version of $\mathrm{k}$-Wave on an NVIDIA Tesla P40 Pascal GPU with 3840 CUDA cores and 24 GB of memory.

The simulations were run in free-field, and with the transducer in two different positions relative to the skull to match the experiments. To enable comparison, the simulated transducer position was defined using the offsets measured in the experiment. For all three case (free-field and two positions within to the skull), simulations were repeated with driving signals of $1,5,10,50,100$, and 500 cycles. For each simulation, the time varying pressure at the position of the peak pressure was recorded as well as the maximum pressure at each grid point in the simulation over the complete time duration.

\section{B. Results}

The focal waveforms for a 500 cycle burst are shown in Fig. 2. Broadly, the simulated results match the experimental results. The primary differences are the longer time period of approximately 800-900 $\mu$ over which undulations in the signal envelope occur, and that the multiple reflections are of slightly higher amplitude relative to the main pulse (data not shown). This may be due to differences in the skull material properties in the simulations compared to the experiments, as well as the noise-floor in the experiments. Slices through the $3 \mathrm{D}$ pressure field are shown in Fig. 3. The complexity of the field pattern increases with the number of cycles as interference creates a more complex sidelobe pattern, as is observed in free-field. The effect of standing waves is to further superimpose oscillations in pressure amplitude onto this pattern, and these appear to be established once the burst length reached 50 cycles.

Consistent with the experiments, the distortions are also greater for Position 2 where the transducer focus is closer to the skull base and the position of the transducer relative to the skull results in a greater range of angles of incidence. In 

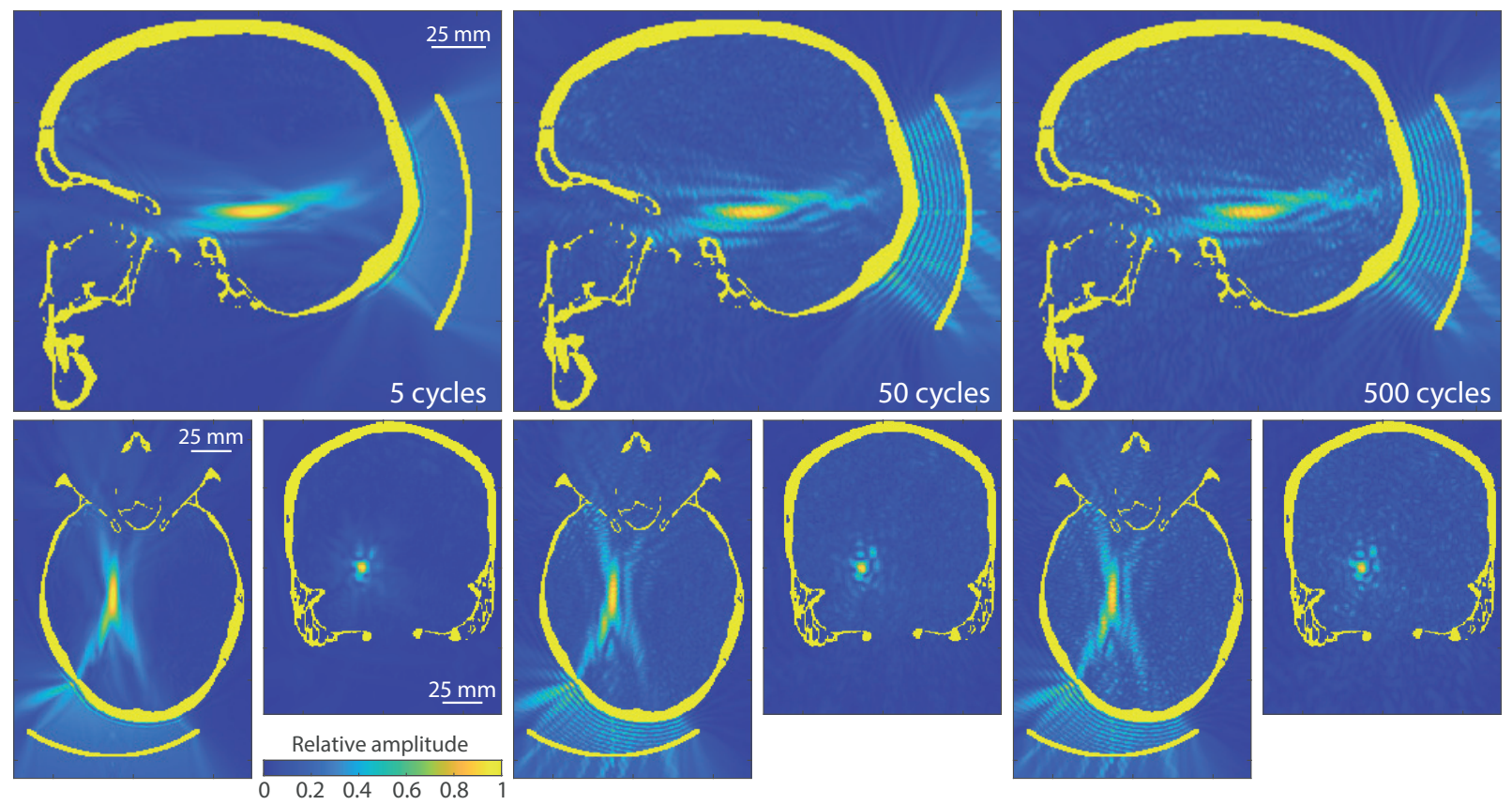

Fig. 3. Simulated acoustic pressure within the skull with the transducer at position 2 , laterally and inferiorly offset from the skull centre with bursts of 5 , 50 and 500 cycles.

general, the amplitudes of the side lobes are greater relative to the focal pressure in the skull compared to free-field. In all cases, the aberration of the field is not great enough to result in focal splitting.

\section{SUMMARY}

Experimental measurements and numerical simulations were conducted to assess the formation of standing waves within the skull when using low frequencies and a large aperture transducer. Standing wave effects were clearly visible in both experiments and simulations once a burst length of 50 cycles or more was employed. However, in general, distortions to the main focal lobe were small and the pattern of sidelobes within the skull was not significantly more complex than the free-field pressure distribution. This preliminary study has demonstrated the suitability of these transducer parameters for targeted BBB opening, and has shown that standing wave effects can be avoided by using burst lengths of less than 50 cycles. This will form the basis of further investigation of the effectiveness of BBB opening using these parameters.

\section{ACKNOWLEDGMENT}

The authors would like to thank Tom Minden, Ian Simcock and team at Great Ormond Street Hospital for CT scans of the skull.

\section{REFERENCES}

[1] E. Konofagou, Y.-S. Tunga, J. Choi, T. Deffieux, B. Baseri, and F. Vlachos, "Ultrasound-induced blood-brain barrier opening," Curr Pharm Biotechnol, vol. 13, no. 7, pp. 1332-1345, 2012.

[2] N. Lipsman, Y. Meng, A. Bethune, Y. Huang, B. Lam, M. Masellis, N. Herrmann, C. Heyn, I. Aubert, A. Boutet, and G. Smith, "Blood-brain barrier opening in alzheimer's disease using mr-guided focused ultrasound," Nat Commun, vol. 9, no. 1, p. 2336, 2018.
[3] N. McDannold, Y. Zhang, J. G. Supko, C. Power, T. Sun, N. Vykhodtseva, A. J. Golby, and D. A. Reardon, "Blood-brain barrier disruption and delivery of irinotecan in a rat model using a clinical transcranial mri-guided focused ultrasound system," Sci Rep, vol. 10, no. 1, p. 8766, 2020.

[4] A. Kyriakou, E. Neufeld, B. Werner, M. M. Paulides, G. Szekely, and N. Kuster, "A review of numerical and experimental compensation techniques for skull-induced phase aberrations in transcranial focused ultrasound," Int J Hyperthermia, vol. 30, no. 1, pp. 36-46, 2014.

[5] J. Song, A. Pulkkinen, Y. Huang, and K. Hynynen, "Investigation of standing-wave formation in a human skull for a clinical prototype of a large-aperture, transcranial mr-guided focused ultrasound (mrgfus) phased array: An experimental and simulation study," IEEE Trans Biomed Eng, vol. 59, no. 2, pp. 435-444, 2012.

[6] S. Morse, A. Pouliopoulos, T. Chan, M. J. Copping, J. Lin, N. J. Long, and J. J. Choi, "Rapid short-pulse ultrasound delivers drugs uniformly across the murine blood-brain barrier with negligible disruption," Radiology, vol. 291, no. 2, pp. 459-466, 2019.

[7] B. E. Treeby, The effect of hair on human sound localisation cues. $\mathrm{PhD}$ thesis, University of Western Australia, 2007.

[8] K. Hynynen and F. Jolesz, "Demonstration of potential noninvasive ultrasound brain therapy through an intact skull," Ultrasound Med Biol, vol. 24, p. 275-283, February 1998.

[9] L. A. Gimeno, E. Martin, O. Wright, and B. E. Treeby, "Experimental assessment of skull aberration and transmission loss at $270 \mathrm{kHz}$ for focused ultrasound stimulation of the primary visual cortex," in IEEE International Ultrasonics Symposium, pp. 556-559, 2019.

[10] B. E. Treeby, J. Jaros, A. P. Rendell, and B. T. Cox, "Modeling nonlinear ultrasound propagation in heterogeneous media with power law absorption using a k-space pseudospectral method," J. Acoust. Soc. Am., vol. 131, no. 6, pp. 4324-4336, 2012.

[11] E. S. Wise, B. T. Cox, J. Jaros, and B. E. Treeby, "Representing arbitrary acoustic source and sensor distributions in fourier collocation methods," J. Acoust. Soc. Am., vol. 146, no. 1, pp. 278-288, 2019.

[12] J. Aubry, M. Tanter, M. Pernot, J. Thomas, and M. Fink, "Experimental demonstration of noninvasive transskull adaptive focusing based on prior computed tomography scans," J. Acoust. Soc. Am., vol. 113, no. 1, pp. 84-93, 2003. 